\title{
Quantitative Analysis of Diffusion Tensor Orientation: Theoretical Framework
}

\author{
Yu-Chien Wu, ${ }^{1,2}$ Aaron S. Field, ${ }^{3}$ Moo K. Chung, ${ }^{2,4,5}$ Benham Badie, ${ }^{6}$ \\ and Andrew L. Alexander ${ }^{1,2,7 *}$
}

\begin{abstract}
Diffusion-tensor MRI (DT-MRI) yields information about the magnitude, anisotropy, and orientation of water diffusion of brain tissues. Although white matter tractography and eigenvector color maps provide visually appealing displays of white matter tract organization, they do not easily lend themselves to quantitative and statistical analysis. In this study, a set of visual and quantitative tools for the investigation of tensor orientations in the human brain was developed. Visual tools included rose diagrams, which are spherical coordinate histograms of the major eigenvector directions, and 3D scatterplots of the major eigenvector angles. A scatter matrix of major eigenvector directions was used to describe the distribution of major eigenvectors in a defined anatomic region. A measure of eigenvector dispersion was developed to describe the degree of eigenvector coherence in the selected region. These tools were used to evaluate directional organization and the interhemispheric symmetry of DT-MRI data in five healthy human brains and two patients with infiltrative diseases of the white matter tracts. In normal anatomical white matter tracts, a high degree of directional coherence and interhemispheric symmetry was observed. The infiltrative diseases appeared to alter the eigenvector properties of affected white matter tracts, showing decreased eigenvector coherence and interhemispheric symmetry. This novel approach distills the rich, 3D information available from the diffusion tensor into a form that lends itself to quantitative analysis and statistical hypothesis testing. Magn Reson Med 52:1146-1155, 2004. () 2004 Wiley-Liss, Inc.
\end{abstract}

Key words: rose diagram; scatter matrix; directional analysis; anisotropy; dyadic tensor; oligodendroglioma

Diffusion-tensor MRI (DT-MRI) provides unique microstructural and physiological information about biological tissues that is not available through other noninvasive means (1). The diffusion tensor represents water molecular diffusivity in the presence of restrictions, such as cellular and cytoplasmic barriers. The anisotropy of the diffusion,

${ }^{1}$ Department of Medical Physics, University of Wisconsin, Madison, Wiscon$\sin$

${ }^{2}$ W.M. Keck Laboratory for Functional Brain Imaging and Behavior, University of Wisconsin, Madison, Wisconsin.

${ }^{3}$ Department of Radiology, University of Wisconsin, Madison, Wisconsin. ${ }^{4}$ Department of Statistics, University of Wisconsin, Madison, Wisconsin.

${ }^{5}$ Department of Biostatistics and Medical Informatics, University of Wisconsin, Madison, Wisconsin.

${ }^{6}$ Departmentof Neurosurgery, University of Wisconsin, Madison, Wisconsin.

${ }^{7}$ Department of Psychiatry, University of Wisconsin, Madison, Wisconsin.

Grant sponsor: National Institutes of Health $(\mathrm{NIH})$; Grant numbers: RO1 EB002012; R21 DA015879; P30 HD03352.

${ }^{\star}$ Correspondence to: Andrew L. Alexander, Ph.D., W.M. Keck Laboratory for Functional Brain Imaging and Behavior Waisman Center, 1500 Highland Ave., University of Wisconsin, Madison, WI 53705-2280. E-mail: alalexander2@facstaff.wisc.edu

Received 29 July 2003; revised 10 June 2004; accepted 10 June 2004.

DOI 10.1002/mrm.20254

Published online in Wiley InterScience (www.interscience.wiley.com).

(c) 2004 Wiley-Liss, Inc. described by the relative anisotropy (RA), the fractional anisotropy (FA), or the volume ratio (VR), is predominantly caused by the orientation of fiber tracts in white matter (WM) and is influenced by intraaxonal organization, density of fiber, and degree of myelination (2). While many recent studies using DT-MRI have focused on ADC and anisotropy measurements (3-6), these studies have not quantified the eigenvector orientation properties of the diffusion tensor in specific anatomic regions, such as WM tracts or basal ganglia, or in specific disease states, such as edema or infiltrative tumor.

RGB (red-green-blue) color maps and WM tractography methods have been developed to display the directional information and tissue organization described by the diffusion tensor (7). However, these methods, although visually appealing, do not lend themselves well to quantitative and statistical analysis, thereby limiting their utility.

The theory and methodology of directional statistics (8) is appropriate for the quantitative analysis of diffusiontensor orientation. Directional statistics is a powerful set of tools for describing the angular distribution of a group of unit vectors on a spherical sample space. Under the assumption that the direction of the major eigenvector of the diffusion tensor is collinear with the direction of WM fibers (9), it is possible to explicitly quantify the distributions of fiber tracts either in a region of interest or for entire brain using directional statistics of the major eigenvectors. The mean orientation and spread of the vector distribution may be used to assess the degree of fiber coherence in a region of interest, the degree of deviation or disruption by a pathologic process, or the interhemispheric symmetry of specific WM tracts.

In this study, a set of methods for visualizing and quantifying the directional statistics of DT eigenvectors in a region of interest are described. These methods are applied to the DT-MRI brain studies of five healthy human subjects. The eigenvector dispersion and interhemispheric symmetry are quantified for several well-characterized fiber tracts. Further, the application of these methods to brain pathology is demonstrated by using them to analyze two varieties of pathologic tract disruption: tumor infiltration and vasogenic edema (one subject with each).

\section{THEORY}

\section{Diffusion Tensor and Major Eigenvector}

In brain tissues, water diffusion can be characterized by the symmetric $3 \times 3$ diffusion tensor matrix $\mathbf{D}$ :

$$
\mathbf{D}=\left[\begin{array}{lll}
\mathrm{D}_{x x} & \mathrm{D}_{x y} & \mathrm{D}_{x z} \\
\mathrm{D}_{y x} & \mathrm{D}_{y y} & \mathrm{D}_{y z} \\
\mathrm{D}_{z x} & \mathrm{D}_{z y} & \mathrm{D}_{z z}
\end{array}\right] .
$$


a

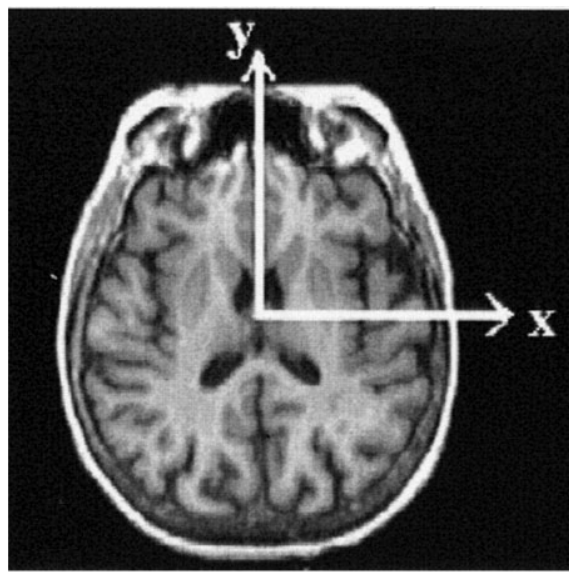

b
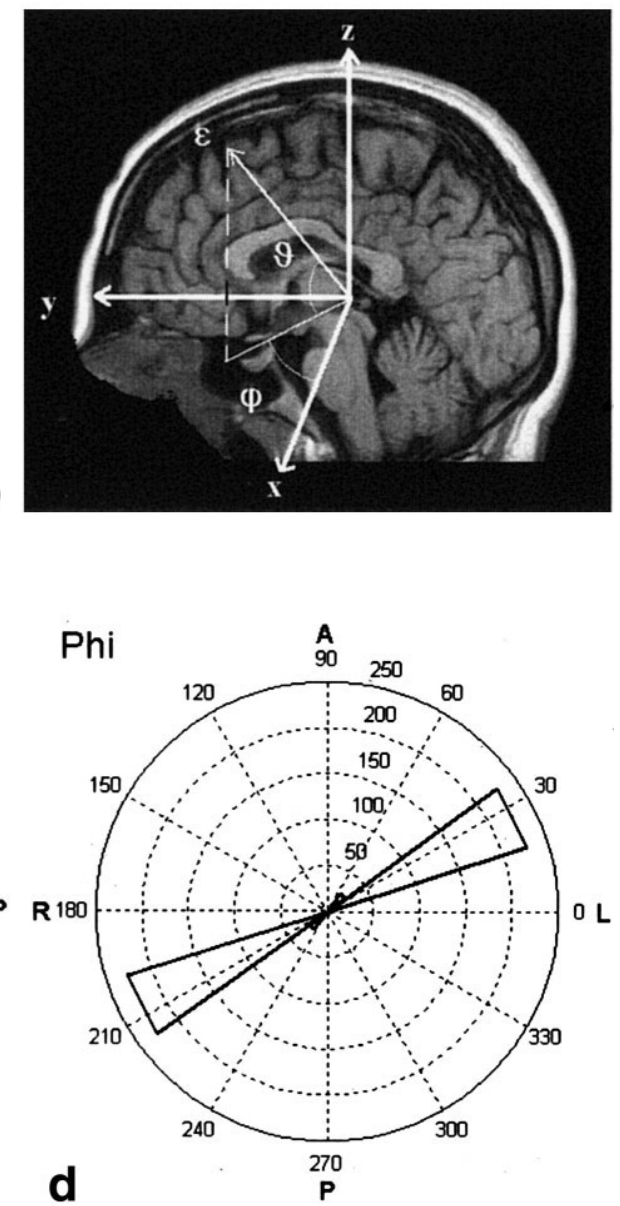

FIG. 1. a,b: The complementary angle of the polar angle $(\Theta)$ denotes the angle between the eigenvector and the $x-y$ plane. The azimuthal angle $(\phi)$ denotes the angle between the $x$-axis and the vector's projection on the $x-y$ plane. The $x, y$, and $z$ axes correspond to left, anterior, and superior, respectively. The rose diagram in theta (c) and phi (d) of an arbitrary distribution having the direction along $\boldsymbol{\varepsilon}$, as drawn in (b).

The diffusion tensor describes the magnitude and orientation of molecular mobility. The tensor can be diagonalized to estimate the major, medium, and minor eigenvalues, $\lambda_{1}$. $\lambda_{2}$, and $\lambda_{3}$, respectively. The corresponding directions of these eigenvalues are the major, medium, and minor eigenvectors, $\boldsymbol{\varepsilon}_{1}, \boldsymbol{\varepsilon}_{2}$, and $\boldsymbol{\varepsilon}_{3}$, respectively. The major eigenvector $\varepsilon_{1}$ associated with $\lambda_{1}$ is typically assumed to be collinear with the orientation of WM fiber tracts in the brain (9).

\section{Eigenvector Distribution Visualization}

As described above, color maps can be used to display directional trends, but such maps are generally not quantitative. A more analytical alternative is to calculate the distribution of major eigenvectors according to their angles, $\Theta$ and $\phi$, in 3D space, where $\Theta$ (complementary angle of the polar angle) denotes the angle between the eigenvector and the $x$-y plane and $\phi$ (azimuthal angle) denotes the angle between the eigenvector's projection in the $x-y$ plane and the $x$-axis. The diffusion tensor eigenvectors in the left and right hemispheres are expected to show mirror symmetry, so the azimuthal angle for the right side of the brain was computed in the opposite sense (e.g., $\phi_{\mathrm{R}}=\pi-$
$\left.\phi_{\mathrm{L}}\right)$. The $x, y$, and $z$ axes correspond to the anatomic directions of left (L), anterior (A), and superior (S), respectively, as shown in Fig. 1a,b. A "left-handed" coordinate convention was chosen because the images were displayed in radiologic coordinates where right and left are reversed. This distribution may be calculated for any region of interest (ROI) and subjected to further analysis. It may also be displayed using plotting tools as a rose diagram in Fig. $1 \mathrm{c}, \mathrm{d}(8)$ or a $3 \mathrm{D}$ angular scatterplot. The rose diagram is a spherical coordinate histogram of either $\Theta$ or $\phi$ in which the area of each sector bin is proportional to the frequency of occurrence for the corresponding range of angles. For the 3D scatterplot, the eigenvector direction of each voxel is represented as a point at $\Theta$ and $\phi$ on the surface of a sphere.

\section{Scatter Matrix}

For a group of unit vectors having a common origin, the tip of each vector is a point on a unit sphere. Consequently, $\boldsymbol{\varepsilon}_{11}, \ldots, \boldsymbol{\varepsilon}_{1 \mathrm{n}}$ are considered to be $\mathrm{n}$ points on the spherical surface. Under this assumption, the dispersion of eigenvectors can be described by the scatter matrix $\mathbf{T}$ about the origin, defined by: 


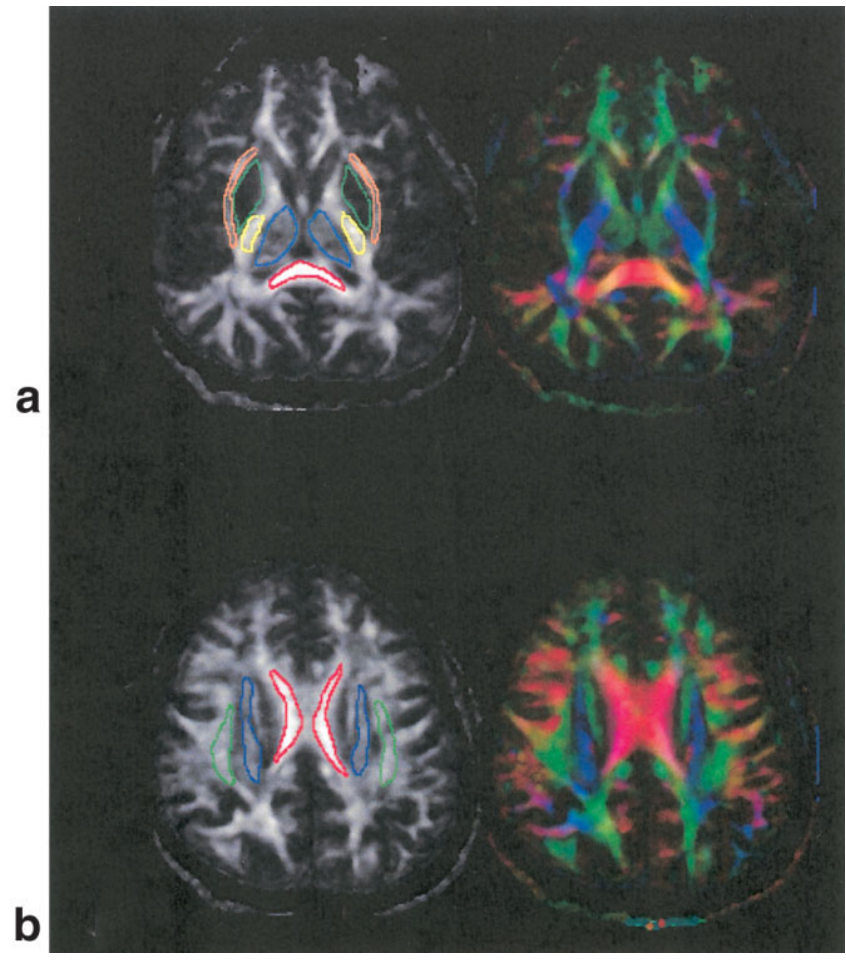

FIG. 2. FA and color maps of one normal healthy subject. The 3D ROls were constructed by $2 \mathrm{D}$ ROls drawn on contiguous axial slices. a: The ROIs included in this FA map are EC (orange), lentiform nucleus (green), PLIC (yellow), thalamus (blue), and callosal splenium (red). b: The ROIs included in this FA map are SLF (green), CR (blue), and CC (red).

$$
\mathbf{T}=\frac{1}{\mathrm{n}} \sum_{\mathrm{i}=1}^{\mathrm{n}} \boldsymbol{\varepsilon}_{\mathrm{li}} \boldsymbol{\varepsilon}_{\mathrm{li}}^{\mathrm{t}}
$$

where $\mathrm{t}$ denotes vector transpose. Like the diffusion tensor, $\mathbf{T}$ is a $3 \times 3$ diagonally symmetric matrix. The eigenvalues $t_{1}, t_{2}$, and $t_{3}$ of the scatter matrix describe the distribution of points in spherical coordinates. In addition, the eigenvectors of the scatter matrix, $\mathbf{t}_{1}, \mathbf{t}_{2}$, and $\mathbf{t}_{3}$, describe the orientation of this distribution. In this application, the unit vectors $\boldsymbol{\varepsilon}_{1 \mathrm{i}}$ are the major eigenvectors of the diffusion tensors (D) for the $\mathrm{i}^{\text {th }}$ voxel within an ROI. The scatter matrix, T, thus quantifies the distribution of major eigenvectors within a specified region.

\section{Anisotropy of the Eigenvector Scatter Matrix}

The angular uniformity of the eigenvector distribution may be described using anisotropy measures of $\mathbf{T}$ that are equivalent to the relative anisotropy (RA) measure described by Basser and Pierpaoli (1) for the diffusion tensor:

$$
\operatorname{raT}=\frac{\sqrt{\left(t_{1}-\bar{t}\right)^{2}+\left(t_{2}-\bar{t}\right)^{2}+\left(t_{3}-\bar{t}\right)^{2}}}{\sqrt{6 \bar{t}}},
$$

where $\bar{t}$ is the mean eigenvalue. In this case, $\bar{t}=0.333$, since the major eigenvectors are unit vectors. This anisotropy measure (raT) is normalized to range between 0 (isotropic) and 1 (completely anisotropic). ROIs with high raT anisotropy indicate regions with highly coherent or collinear eigenvector organization, whereas regions with low anisotropy indicate areas where the distribution of eigenvectors is either random or highly noncollinear. a

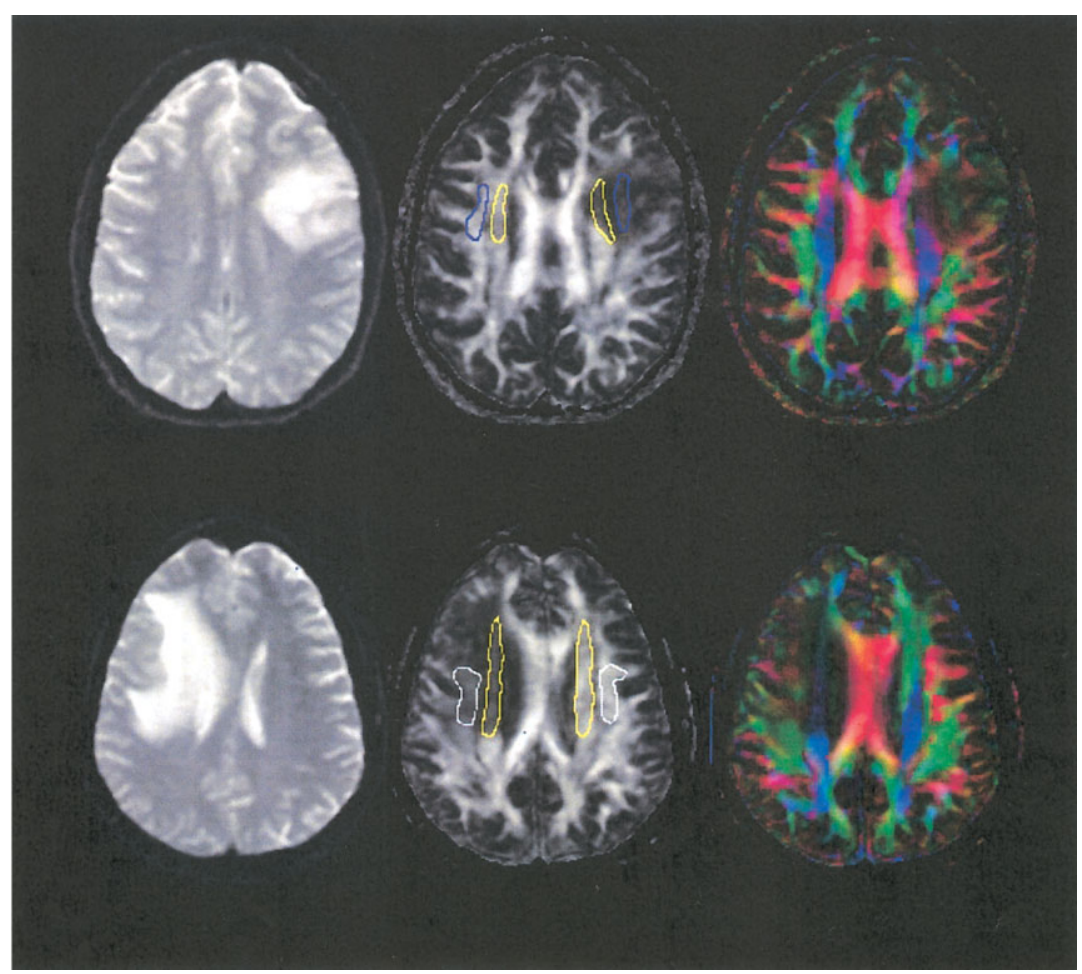

b
FIG. 3. ADC, FA, and color maps of the oligodendroglioma and the vasogenic edema subjects. a: Both the left corona radiata (yellow ROI) and superior longitudinal fasciculus (blue ROI) were infiltrated by the oligodendroglioma. The oligodendroglioma has hyperintensity on the ADC map. However, the FA and color maps show decreasing intensity of the WM fibers affected by this tumor. b: Both the right corona radiata (yellow ROI) and superior longitudinal fasciculus (white ROI) were infiltrated by the vasogenic edema. Similar to the oligodendroglioma, edema shows hyperintensity on the ADC map, but hypointensity on the FA and color maps. Contralateral ROls are also shown on the FA maps for both cases. 


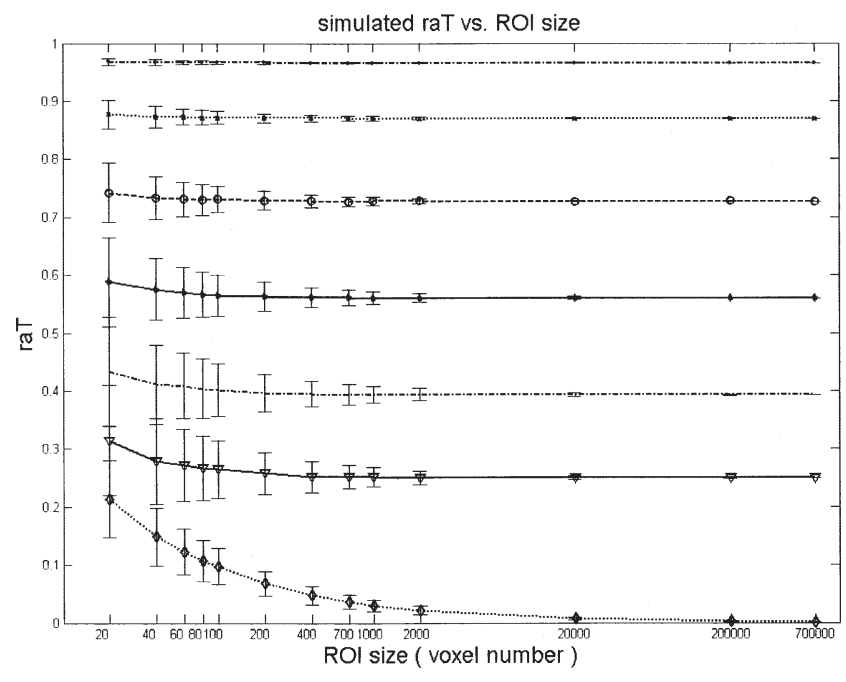

FIG. 4. Monte Carlo computer simulation of the effect of ROI size on the directional analysis. Eigenvector fields were generated at seven levels of raT. The SD and mean raT were calculated from 1000 random samples. The overestimation of raT is greater for more isotropic eigenvector distributions. The SD of estimated raT values is similarly a function of the inherent raT and voxel number.

\section{Symmetry Index}

A measure of interhemispheric symmetry for specific brain regions may be calculated using a symmetry index:

$$
S=\mathbf{t}_{1 \mathrm{~L}} \cdot \mathbf{t}_{1 \mathrm{R}},
$$

which is the dot product of the scatter matrix major eigenvectors of the left and right hemisphere ROIs, $\mathbf{t}_{1 \mathrm{~L}}$ and $\mathbf{t}_{1 \mathrm{R}}$. An $\mathrm{S}$ value approaching unity indicates a high degree of symmetry, while $S$ near zero indicates that the scatter matrixes are nearly orthogonal.

\section{SUBJECTS AND METHODS}

Five healthy volunteers (three men, two women), ages 18-22 years, participated in the study in compliance with the university guidelines for human subjects research. DTMRI studies were performed at $3 \mathrm{~T}$ using a peripheralpulse gated, single-shot, diffusion-weighted spin echo EPI sequence with diffusion tensor encoding. A quadrature head coil was used for RF excitation and reception. Twelve diffusion encoding directions were selected using a numerically optimized, minimum energy encoding scheme (10). The diffusion gradient pulse width $(\delta)$, the separation between the diffusion gradient onsets $(\Delta)$, and the gradient amplitude were $21 \mathrm{~ms}, 27 \mathrm{~ms}$, and $30 \mathrm{mT} / \mathrm{m}$, respectively, generating a diffusion weighting of $912 \mathrm{~s} /$ $\mathrm{mm}^{2}$ in each direction. Thirty-nine contiguous, axial, 3-mm thick slices covering the entire cerebrum and part of the cerebellum were obtained in 6.5-8 $\mathrm{min}$, dependant upon the subjects heart rate. The other imaging parameters were $\mathrm{T}_{\mathrm{R}} / \mathrm{T}_{\mathrm{E}}=4000 / 72 \mathrm{~ms}, 240 \mathrm{~mm}$ field of view, $120 \times$ 120 acquisition matrix interpolated to $256 \times 256$.

To investigate the possible use of directional statistics in a clinical application, the DT-MRI data from two male subjects with brain neoplasms (one oligodendroglioma Grade II, one metastatic brain tumor) were examined prior to surgical resection. These DT-MRI studies were performed in a $1.5 \mathrm{~T}$ scanner using a similar pulse sequence (although not pulse gated) and 23 diffusion-encoding directions. The 23 encoding directions were selected using a numerically optimized, minimum energy encoding scheme (10). The diffusion gradient pulse width, separation, and amplitude were $16 \mathrm{~ms}$, $21 \mathrm{~ms}$, and $40 \mathrm{mT} / \mathrm{m}$, respectively, generating a diffusion weighting of $995 \mathrm{~s} / \mathrm{mm}^{2}$ in each direction. Other imaging parameters were $\mathrm{T}_{\mathrm{R}} / \mathrm{T}_{\mathrm{E}}=4000 / 72 \mathrm{~ms}$, and four NEX. Thirtynine axial, 3-mm thick, contiguous slices covering whole cerebrum with a $240 \mathrm{~mm}$ FOV and $128 \times 128$ matrix (interpolated to $256 \times 256$ ), were obtained in roughly $15 \mathrm{~min}$. In these two cases, the corona radiata and superior longitudinal fasciculus were infiltrated by the oligodendroglioma in one patient and by vasogenic edema in the other patient. Informed consent was obtained from all subjects in compliance with the university guidelines for human subjects research.

Distortions in the diffusion-weighted images from eddy currents were corrected using affine image registration software (11). Directional apparent diffusivities

Table 1

Mean raT, Symmetry Index S, Size and Maximum Variation of raT of Each ROI across Five Normal Subjects

\begin{tabular}{lccccc}
\hline ROI & raT & S & Voxel numbers & min_vol_no & max_dev_raT \\
\hline CC $^{a}$ & $0.94 \pm 0.02$ & $0.99 \pm 0.01$ & $357.50 \pm 99.52$ & 262 & $<0.002$ \\
PLIC & $0.87 \pm 0.03$ & $0.98 \pm 0.02$ & $304.40 \pm 67.00$ & 217 & $<.008$ \\
SLF & $0.85 \pm 0.05$ & $0.98 \pm 0.01$ & $346.00 \pm 122.90$ & 178 & $<0.010$ \\
CR & $0.80 \pm 0.04$ & $0.98 \pm 0.01$ & $1111.30 \pm 242.10$ & 707 & $<0.006$ \\
CC & $0.78 \pm 0.04$ & - & $566.70 \pm 204.64$ & 287 & $<0.009$ \\
EC & $0.57 \pm 0.07$ & $0.99 \pm 0.00$ & $276.40 \pm 55.78$ & 182 & $<0.026$ \\
Th & $0.40 \pm 0.03$ & $0.99 \pm 0.01$ & $1470.00 \pm 205.39$ & 524 & $<0.014$ \\
LN & $0.37 \pm 0.07$ & $0.88 \pm 0.13$ & $928.80 \pm 246.17$ & 1189 & $<0.020$ \\
WM $^{\text {b }}$ & $0.12 \pm 0.02$ & - & $152890.00 \pm 20495$ & 297357 & $<0.002$ \\
WB $^{\text {d }}$ & $0.11 \pm 0.01$ & - & $319510.00 \pm 34338$ & $<0.002$ & $<$
\end{tabular}

The uncertainty after the \pm sign is 1 SD across five healthy subjects. The min_vol_no stands for the minimum ROI size (voxel number) in the brains of five healthy subjects. The maximum deviation of raT, max_dev_raT, due to the ROI size and raT level, was estimated from Monte Carlo simulation (Fig. 4) using the information of min_vol_no and raT value of each ROI.

PLIC: posterior limb of internal capsule; SLF: superior longitudinal fasciculus; EC: external capsule; CR: corona radiata; CC: corpus callosum; Th: thalamus; LN: lentiform nucleus; ${ }^{a} \mathrm{CC}$ : ROI on left and right corpus callosum body; ${ }^{\mathrm{b}} \mathrm{CC}$ : ROI on callosal genu and splenium; 'WM: 3D white matter ROI used FA values between 0.3 and 1.0; dWB: 3D whole brain ROI used ADC values between 500 and $1000 \mu \mathrm{m}^{2} / \mathrm{sec}$. 

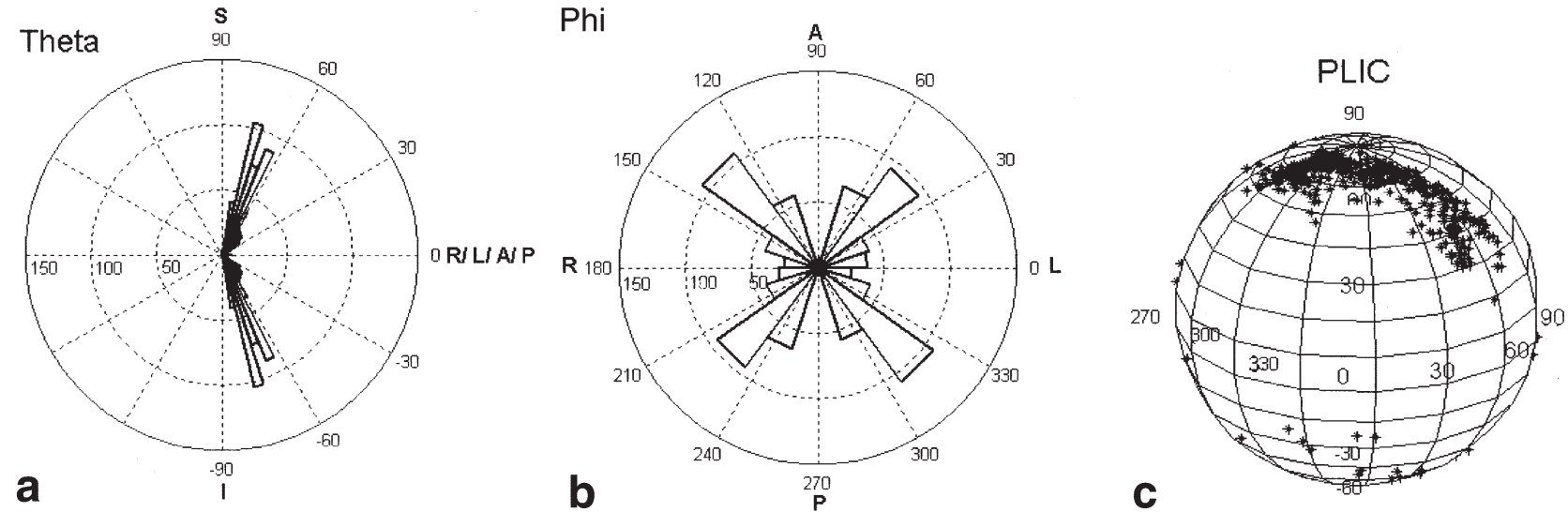

FIG. 5. Rose diagrams and 3D scatterplot of major eigenvectors for the left posterior limb of internal capsule (PLIC) of one healthy volunteer. a: Rose diagram of angle $\Theta$. The distribution of eigenvector $\Theta$ angles is unimodal. b: Rose diagram of azimuthal angle $(\phi)$. c: 3D scatterplot of $\Theta$ vs. $\phi$. These diagrams explicitly quantify the primary superior-inferior orientation of fibers in the PLIC. S-I: superior-inferior, A-P: anterior-posterior, and L-R: left-right.

were calculated by the log ratio of the nondiffusionweighted signal to the diffusion-weighted signal divided by the diffusion weighting factor. The diffusion tensor for each voxel location was estimated using a linear least squares fit to the registered directional diffusivities (10). The diffusion tensor was diagonalized (12) to estimate the eigenvalues and eigenvectors at each voxel location. $3 \mathrm{D}$ maps of the trace of the diffusion tensor, trace $\{\mathbf{D}\}$, and the fractional anisotropy, FA, were generated. Color RGB maps of the major eigenvector direction weighted by FA were generated for WM tract identification and segmentaion (7).

Three-dimensional brain ROIs were selected on the FA maps. For the five healthy subjects, the regions included the posterior limb of internal capsule (PLIC), external capsule (EC), corona radiata (CR), corpus callosum (CC), callosal genu and splenium, superior longitudinal fasciculus (SLF), thalamus (Th), and lentiform nucleus (LN) (see Fig. 2 for example). In the patients with brain tumors, symmetric regions in the corona radiata and superior longitudinal fasciculus were selected (Fig. 3). ROIs were selected bilaterally for each structure to test interhemispheric symmetry. The 3D ROIs were constructed by 2D ROIs drawn on contiguous axial slices. Whole brain (combined gray matter (GM) and white matter) scatter matrix calculation was done by segmenting the CSF signal out using empirically derived ADC threshold in the range of 500-1000 $\mathrm{mm}^{2} / \mathrm{sec}$. Whole brain WM selection was done by selecting voxels with FA values greater than 0.3 .

For each voxel within an ROI, the major eigenvector of the diffusion tensor was recorded. Directional vector analysis was applied to each anatomical region (ROI) of major eigenvectors. The scatter matrix (T), the anisotropy (raT), and the symmetry index (S) were calculated according to Eqs. 2-4. Because the sign of the eigenvectors is ambiguous $\left(\varepsilon_{1 \mathrm{i}}\right.$ and $-\varepsilon_{1 \mathrm{i}}$ were considered identical), the analyses included both $\varepsilon_{1 \mathrm{i}}$ and $-\varepsilon_{1 \mathrm{i}}$.

The number of measurements (e.g., voxels in an ROI) may influence the directional statistics. For example, it is likely that for small ROIs in regions with more isotropic
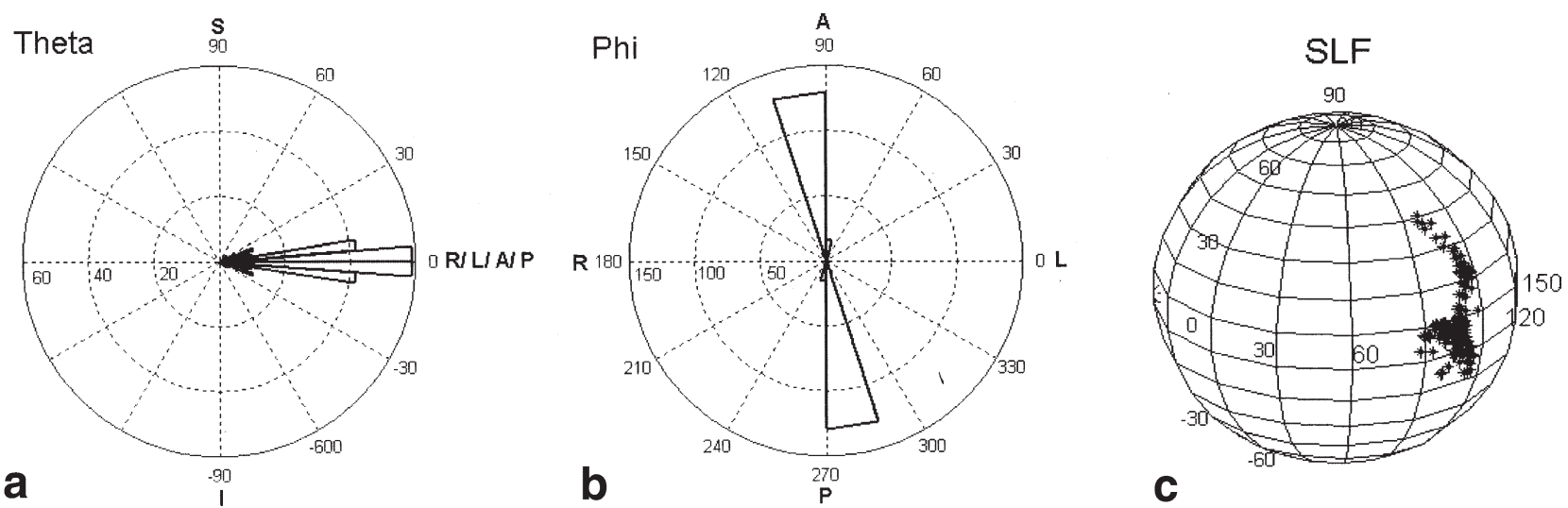

FIG. 6. Rose diagrams and 3D scatterplot for right superior longitudinal fasciculus (SLF) of the same volunteer. a: Rose diagram of angle $\Theta$. Most of the eigenvectors lay close to the $x-y$ plane. b: Rose diagram of azimuthal angle $(\phi)$. The majority of the vectors are oriented anteroposteriorly. c: 3D scatterplot of $\Theta$ vs. $\phi$. These diagrams explicitly quantify the primarily anteroposterior orientation of fibers in this portion of the SLF. 


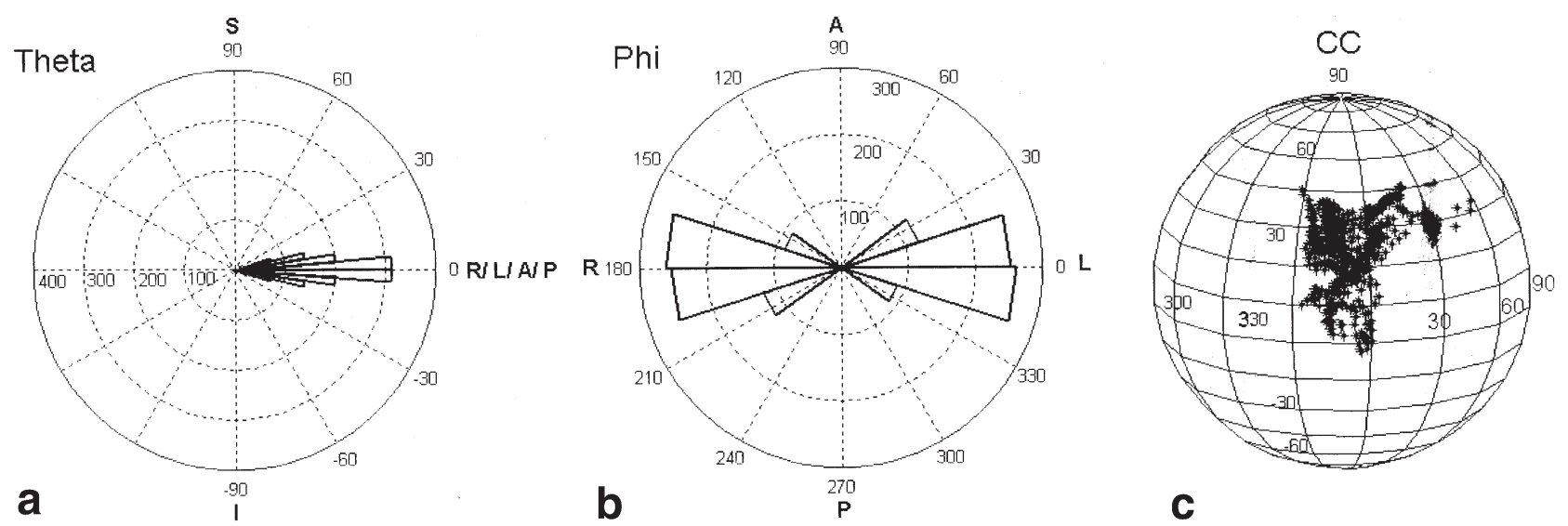

FIG. 7. Rose diagrams and 3D scatterplot for the callosal genu of the same volunteer. a: Rose diagram of angle $\Theta$. Most of the eigenvectors lay close to the $x-y$ plane. b: Rose diagram of azimuthal angle $(\phi)$. The majority of the vectors are left-right oriented. c: 3D scatterplot of $\Theta$ vs. $\phi$. These diagrams explicitly quantify the primarily left-right orientation of fibers in the midsagittal portion of the callosal genu.

eigenvector distributions, the anisotropy of the scatter matrix (raT) will be overestimated with larger variances. A Monte Carlo computer simulation was used to investigate the effect of ROI size (voxel number) on the directional analysis. Eigenvector fields (with $7 \times 10^{6}$ voxels) were generated for seven levels of raT in the absence of noise. Twelve ROI sizes between 20 and $2 \times 10^{5}$ voxels were simulated by randomly sampling from the larger eigenvector field. The random sampling was repeated 1000 times. The raT was calculated for each iteration. The mean and standard deviation (SD) of the measured raT values were calculated for each true raT level and ROI size.

\section{RESULTS}

The effects of ROI size on raT measurements studied with Monte Carlo simulation are shown in Fig. 4. For small ROI sizes, the raT tends to be overestimated. The overestimation of raT is greater for more isotropic eigenvector distributions. The SD of estimated raT values is similarly a function of the inherent raT and voxel number. Both the bias and variance of the estimated raT values decrease if either the sample number is increased or the inherent raT is large. Table 1 lists the average raT values and minimum ROI sizes for the five healthy subjects, along with the corresponding maximum SD of raT estimated from our Monte Carlo simulation. In this study, the maximum simulated deviation of raT is 0.026 (in the external capsule (EC) of one subject with an ROI size of 182 voxels and raT 0.55). The estimated deviation of raT at this level would not have a significant impact on the raT data analysis in this study. Note that the simulation did not take the effects of measurement noise into account. Measurement noise would likely increase the variance of the raT estimates.

The distributions of the major eigenvector directions for left posterior limb of internal capsule, right superior longitudinal fasciculus, and callosal genu of one volunteer are plotted in Figs. 5, 6, and 7, respectively. The rose diagrams of the same ROIs (PLIC, SLF, and CC) for both hemispheres and across all five volunteers are very similar. These three WM fiber tracts are known for their highly organized structure and their orientations in S-I, A-P, and L-R directions, respectively. Our visualization tools clearly depict the distribution of WM tract directions for these WM structures. Figure 8 shows the eigenvector distribution for WM over the whole brain for one subject. The distribution over the whole brain appears much more isotropic, which is consistent with the wide directional distribution of WM tracts in the human brain.

The average eigenvalues of the scatter matrix for the healthy subjects are listed in Table 2. The uncertainty is defined as 1 SD calculated from the estimates across the five subjects and both hemispheres. Table 1 lists the regional average and SD across subjects for the scatter matrix anisotropy, raT, symmetry index, S, and voxel number (ROI size). The CC, PLIC, SLF, and CR demonstrated the most anisotropic eigenvector distributions (i.e., highest raT values) (Table 1). High raT values reflect that most of the major eigenvectors in a selected region are nearly parallel. The major eigenvectors of the EC also appear to show highly anisotropic eigenvector distributions. The smaller scatter matrix anisotropy for EC relative to PLIC or CC is consistent with its divergent organization. The observed scatter matrix anisotropy in the thalamus (Th) and the lentiform nucleus (LN) are lower than most WM regions but are still considerably anisotropic, indicating some degree of coherent organization. The eigenvector scatter matrices for both the whole brain (WB: both GM and WM) and the whole brain WM show more isotropic distributions of eigenvectors, as expected.

The rose diagrams and the 3D scatterplots for eigenvector distributions of PLIC, SLF, EC, CR, and Th are very similar between hemispheres within subjects. This is reflected in the measured mean symmetry index (Table 1). The degree of symmetry was quite high $(\geq 0.98)$ for all of the WM tracts and the thalamus but was lower and more variable across subjects for the lentiform nucleus.

Rose diagrams for the tumor-infiltrated WM and contralateral unaffected WM for the subject with the oligodendroglioma are shown in Fig. 9a-d. The 3D ROIs were drawn bilaterally on the SLF (Fig. 3a: blue ROI). The 2D scatterplot of bilateral eigenvectors is shown in Fig. 9e,f. Rose and 2D 

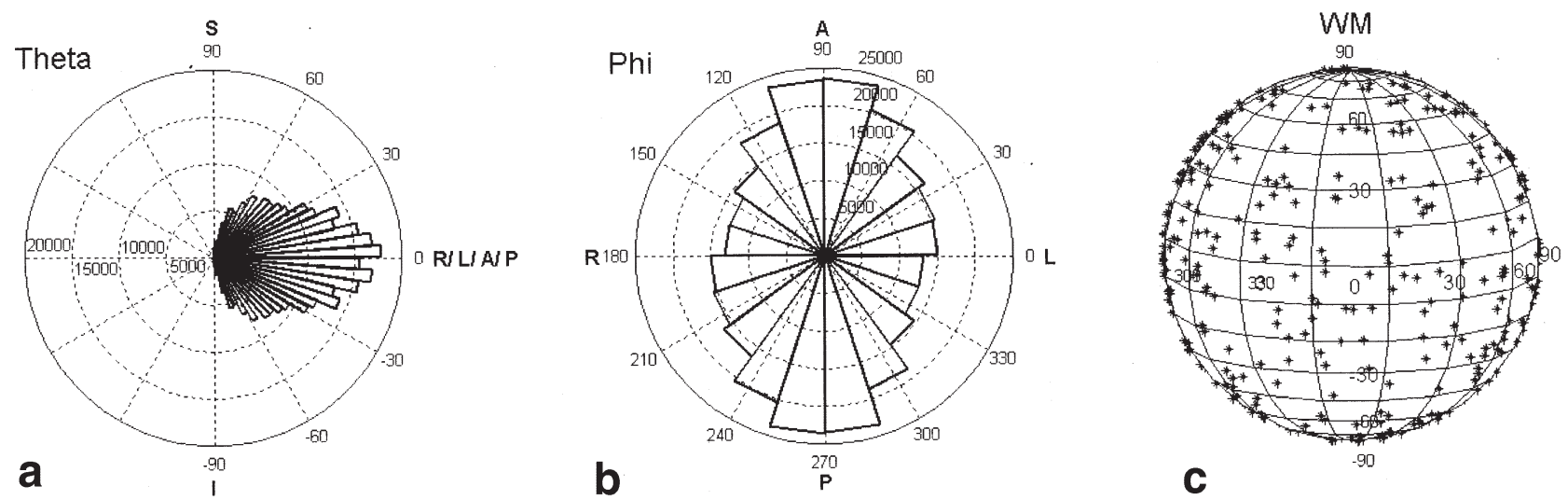

FIG. 8. Rose diagram and 3D scatter plot of whole brain WM using FA thresholds of $>0.3$. This dataset represents the whole brain WM voxels without left or right hemispheres separation and flipping of the eigenvectors over one hemisphere. Because the sign of the eigenvectors is ambiguous, for each $\varepsilon_{1 \mathrm{i}}$ we added $-\varepsilon_{1 \mathrm{i}}$ for more intuitive visual presentation. a: Rose diagram of angle $\Theta$. b: Rose diagram of azimuthal angle $(\phi)$. c: 3D scatterplot of $\Theta$ vs. $\phi$. The distribution of whole brain WM is nearly isotropic due to the wide variety of differently oriented fiber tracts.

scatterplots of the CR for the patient with infiltrating vasogenic edema are shown in Fig. 10 (Fig 3b: yellow ROI). These figures indicate that the tumor infiltration significantly altered the distributions of eigenvectors compared to the contralateral side, whereas the edema infiltration caused fewer changes to the WM eigenvector distributions.

Table 3 lists the symmetry index, raT, and other properties of the scatter matrices for the CR and SLF tracts of both the subject with the infiltrating oligodendroglioma and the subject with the infiltrating vasogenic edema. The SLF infiltration with vasogenic edema showed a decreased raT value. The other tracts that were studied showed less significant changes in raT between affected and unaffected WM regions. However, the symmetry indices of both CR and SLF in the vasogenic edema case are higher than those of the oligodendroglioma subject. This may reflect that the tumor affected WM tract organization more than edema (13-15).

\section{DISCUSSION}

In this study, a set of quantitative and visual tools for analyzing the distribution of diffusion-tensor major eigenvectors in specific anatomic regions was described and tested in both normal subjects and patients with pathology. To our knowledge, a quantitative method for quantifying regional diffusion-tensor eigenvector properties has not been reported previously.

The spherical coordinate for rose diagrams and scatterplots in this study is based on the MRI scanner frame. Slight changes in head position will change the average direction and may affect the hemispheric symmetry. Although head positioning in this study showed excellent symmetry about the midline, no attempt was made to correct for head tilt about the $x$-axis (e.g., the chin moving up or down). In this study, the main comparisons were within-subject (e.g., across hemispheres), so that head tilt about $x$-axis was not a problem. Future studies that use these methods to compare subject groups may want to establish a normalized reference frame, such as the AC-PC line (anterior commissure and posterior commissure) and the brain midline. Note that the coordinate system will not influence the scatter anisotropy measure, raT, as it is directionally invariant.

The size, shape, and orientation of both the voxels and the ROI may also influence the distribution of eigenvectors used to estimate the scatter matrix. If the directional statistics are calculated for a tensor field with heterogeneity in the major eigenvector orientations, the scatter matrix could vary significantly according to the spatial dimensions and orientation of the voxels. A similar problem arises in any imaging study that uses ROIs. With the exception of the whole brain measurements, ROIs were selected in relatively homogeneous WM regions, so that these effects should be minimized. Consequently, in this study, which used anisotropic voxel dimensions $(0.9375 \times$ $0.9375 \times 3$ ), we would expect the results in most of these regions to be relatively independent of the voxel geometry. Conversely, the directional statistics of the whole brain,

Table 2

Eigenvalues of Scatter Matrix T vs. Different ROI

\begin{tabular}{lccc}
\hline \multirow{2}{*}{ ROI } & \multicolumn{3}{c}{$\mathbf{T}$} \\
\cline { 2 - 4 } & $\mathrm{t}_{1}$ & $\mathrm{t}_{2}$ & $\mathrm{t}_{3}$ \\
\hline CC $^{\mathrm{a}}$ & $0.96 \pm 0.02$ & $0.03 \pm 0.01$ & $0.02 \pm 0.01$ \\
PLIC & $0.90 \pm 0.04$ & $0.08 \pm 0.08$ & $0.01 \pm 0.01$ \\
SLF & $0.90 \pm 0.04$ & $0.07 \pm 0.02$ & $0.03 \pm 0.01$ \\
CR & $0.90 \pm 0.04$ & $0.10 \pm 0.02$ & $0.10 \pm 0.02$ \\
CC & $0.85 \pm 0.03$ & $0.13 \pm 0.04$ & $0.02 \pm 0.02$ \\
EC & $0.66 \pm 0.05$ & $0.29 \pm 0.08$ & $0.03 \pm 0.02$ \\
Th & $0.60 \pm 0.03$ & $0.30 \pm 0.04$ & $0.10 \pm 0.01$ \\
LN & $0.52 \pm 0.05$ & $0.33 \pm 0.03$ & $0.11 \pm 0.04$ \\
WM $^{\mathrm{c}}$ & $0.41 \pm 0.02$ & $0.32 \pm 0.02$ & $0.27 \pm 0.02$ \\
WB $^{\mathrm{d}}$ & $0.38 \pm 0.00$ & $0.35 \pm 0.01$ & $0.26 \pm 0.01$
\end{tabular}

$t_{1}, t_{2}$, and $t_{3}$ are the major, medium, and minor eigenvalues of scatter matrix $\mathbf{T}$.

${ }^{\mathrm{a}} \mathrm{CC}$ : $\mathrm{ROI}$ on left and right corpus callosum body; ${ }^{\mathrm{b}} \mathrm{CC}$ : $\mathrm{ROI}$ on callosal genu and splenium; 'WM: 3D white matter ROI used FA values between 0.3 and 1.0; ${ }^{\mathrm{d} W B}$ : $3 \mathrm{D}$ whole brain ROI used ADC values between 500 and $1000 \mu \mathrm{m}^{2} / \mathrm{sec}$. 
FIG. 9. Rose diagrams of the superior longitudinal fasciculus (SLF) for the oligodendroglioma subject. a,b: The distributions of angle $\Theta$ for contralateral and oligodendroglioma invasive SLF. c,d: The distribution of angle $\phi$ for SLF. e,f: The 2D scatterplots. These plots reveal an altered distribution of eigenvectors in the tumor-infiltrated tracts compared to the normal contralateral tracts. 2D scatterplots were used here because of the broad directional distributions that were difficult to view on a single projection of a sphere surface.
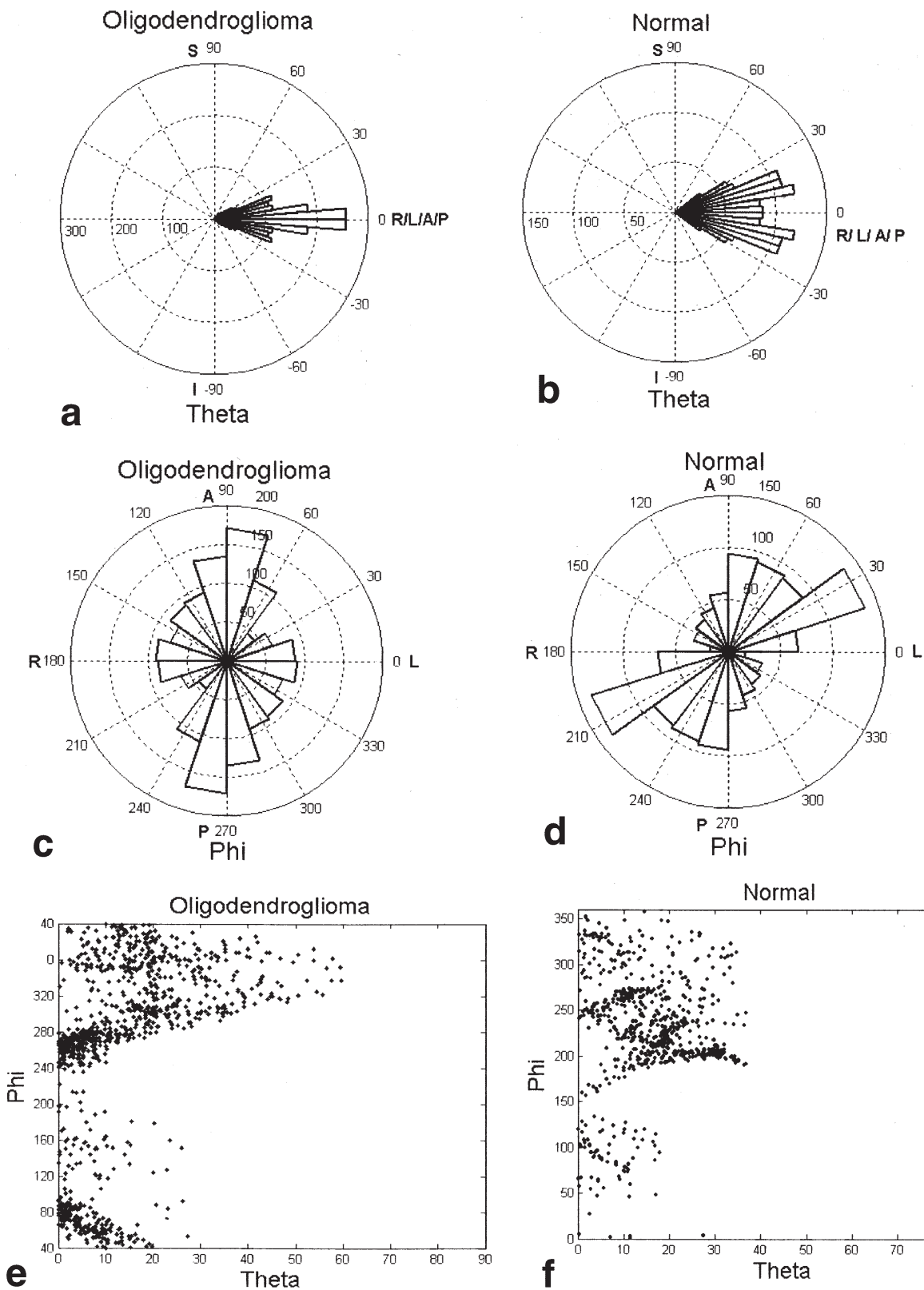

which has substantial heterogeneity of eigenvector orientations, may be much more sensitive to the dimensions and orientations of the sample voxels.

The simulation investigating the effects of ROI size vs. raT measure suggests that the ROI sizes used in this study should not affect the accuracy of the raT measure. While some WM fiber tracts tend to have smaller ROI sizes (e.g., the internal capsule), relatively high raT values minimize the variation. The deep GM structures that we examined, thalamus and lentiform nucleus, have moderate raT value and ROI size around 1000 voxels, which could induce variances in raT at similar levels as that of EC. Although the whole brain tissue and whole brain WM have nearly isotropic characteristics ( $\mathrm{raT}=0.12$ and 0.11 , respectively), the ROI size is very large, with voxel numbers on the order of $10^{5}$. The results of this study indicate that directional statistics can provide meaningful and accurate quantitative information regarding the distribution of eigenvectors in a brain region.

The scatter matrix (also called the dyadic tensor (1618)) has been used previously to study eigenvector dispersion in diffusion-tensor imaging. However, these studies used scatter matrices to describe angular dispersion vs. measurement noise (16) or as a measure of eigenvector coherence across subjects in a normalized image space (18). The scatter matrix approach to quantify the orientation, coherence, and interhemispheric symmetry of regional eigenvector distributions for purposes of tissue characterization is demonstrated for the first time in this study. In recent studies, Li (19) and Hasan et al. (20) estimated the distributions of the polar and azimuthal angles of the diffusion-tensor major eigenvectors in normal brain, but directional statistics were not used. Note that although this study focused on characterizing the angular 

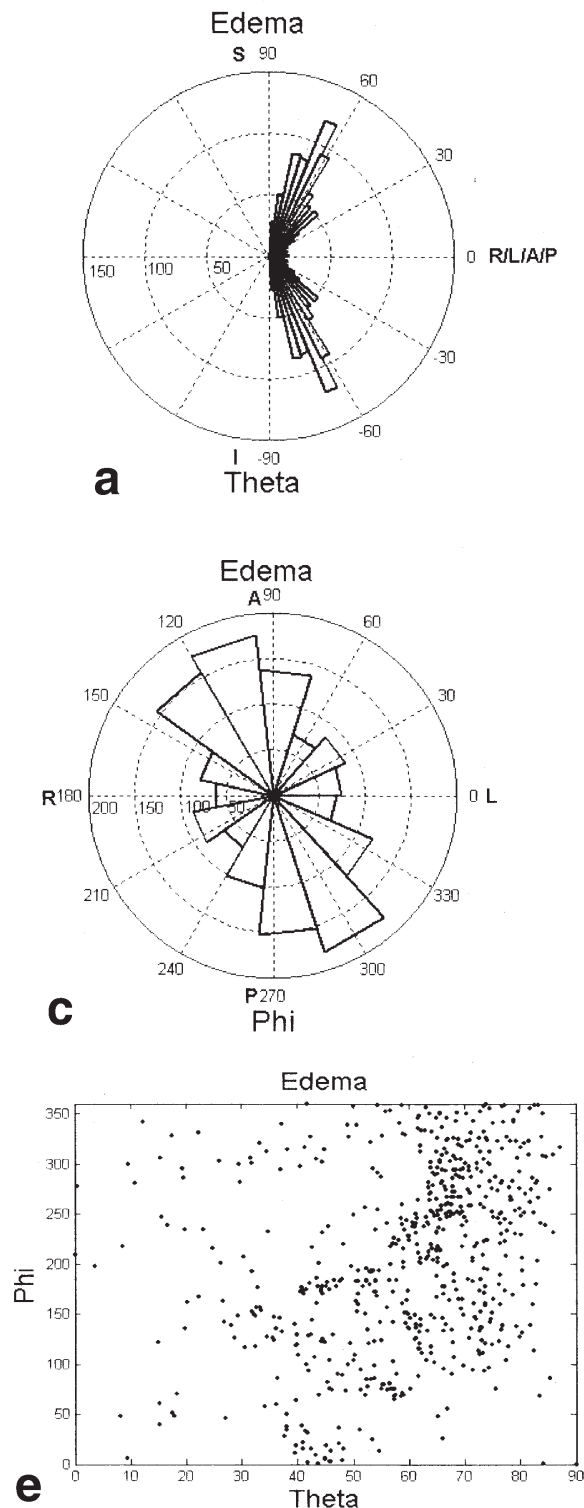
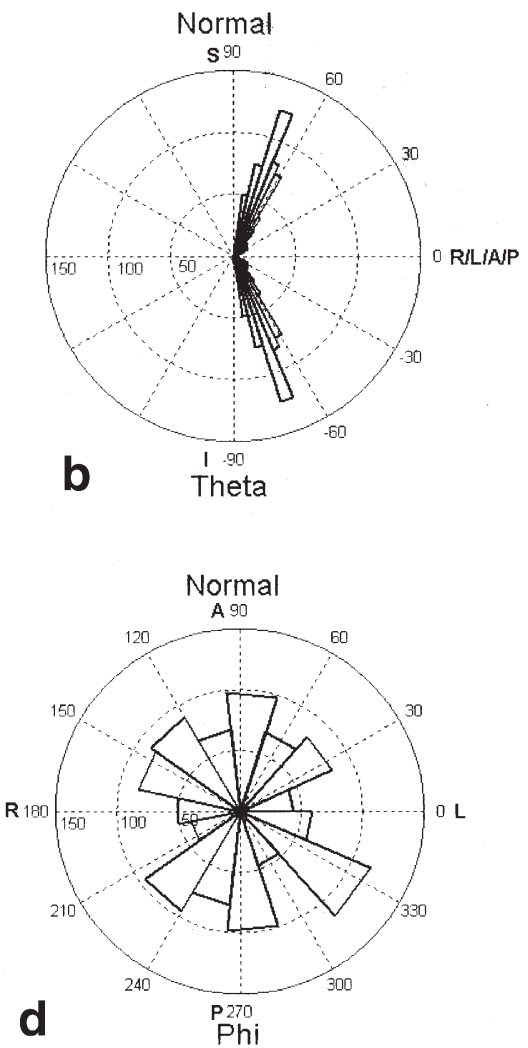

FIG. 10. Rose diagrams of the corona radiata $(\mathrm{CR})$ for the vasogenic edema subject. a,b: The distributions of angle $\theta$ for contralateral normal and edema infiltrating CR. c,d: The distribution of angle $(\phi)$ for CR. e,f: The 2D scatterplots. These plots reveal an altered distribution of eigenvectors in the edema-infiltrated tracts compared to the normal contralateral tracts. distributions of major eigenvectors, the same principles could be applied to the directions of the medium and minor eigenvectors. The distributions of these vectors has been largely ignored in most DT-MRI studies.

As expected, a high degree of eigenvector symmetry was found for all major WM tracts and the thalamus. Although the number of subjects in this study is relatively small, it is expected that the results from other healthy subjects will be similar and that the symmetry index defined herein will be a useful parameter in the assessment of regional WM pathology.

One interesting result is that such deep GM regions as thalamus and lentiform nucleus have moderate raT values. This result in thalamus is consistent with the bowtie plots of eigenvectors as described by Jones et al. (18). Their

Table 3

Scatter Matrix Properties of One Oligodendroglioma Subject and One Edema Subject

\begin{tabular}{|c|c|c|c|c|c|c|}
\hline Subject & $\mathrm{ROI}$ & Side & $\theta$ & $\phi$ & raT & S \\
\hline \multirow{4}{*}{ Oligodendroglioma } & CR & Oligo & 38.32 & 162.95 & 0.74 & 0.80 \\
\hline & & Normal & 73.74 & 162.26 & 0.66 & \\
\hline & SLF & Oligo & 7.47 & 274.62 & 0.46 & 0.74 \\
\hline & & Normal & 17.46 & 307.35 & 0.52 & \\
\hline \multirow[t]{4}{*}{ Vasogenic Edema } & CR & Edema & 87.22 & 166.28 & 0.52 & 0.99 \\
\hline & & Normal & 78.54 & 115.35 & 0.71 & \\
\hline & SLF & Edema & -9.21 & 142.33 & 0.55 & 0.99 \\
\hline & & Normal & -16.86 & 142.65 & 0.73 & \\
\hline
\end{tabular}


study in 10 healthy subjects demonstrated that the thalamus has fairly high "intersubject" coherence, despite a low "intrasubject" fractional anisotropy of the diffusion tensor. However, the results here focus on "intrasubject" properties. One possible explanation of the high hemispheric symmetry of principal eigenvectors in the thalamus may be attributed to the ordered neuroanatomic structure of thalamus, which includes not only major GM nuclei, but also many connecting fibers.

The symmetry index, $\mathrm{S}$, is most meaningful if the angular distributions are both unimodal and symmetric. As the distributions of eigenvectors in a region become more complex, using $\mathbf{t}_{1}$ to represent the main orientation of the distribution may not be appropriate. Further, other measures such as the mode and median vectors (similar to the modes and medians of tensors in Jones et al. (18)) may be preferable to the $\mathbf{t}_{1}$ major eigenvector for symmetry analyses.

As a first step towards clinical application, we identified and quantified increased alteration of eigenvector directional distributions in a case of infiltrating oligodendroglioma as compared to a case of vasogenic edema. It should be noted that these two cases were not differentiated from each other by ADC, FA (15), or conventional MRI, including $T_{2}$-weighted images. There was no obvious bulk mass displacement to account for the altered distribution of eigenvector directions in the oligodendroglioma, thus the shifted phi distribution from the contralateral normal tissue in Fig. 9c,d appears to have resulted from microstructural changes. Therefore, the findings suggest that disruption of tissue microstructure by the infiltrating tumor may differ by nature or degree from that caused by the vasogenic edema, and that this difference may be quantifiable through diffusion-tensor directional statistics. Certainly, no conclusions can be drawn from this small demonstration, but it does suggest that further study of this approach is warranted.

It is unclear whether or not the assumption of collinearity between the major eigenvector and the orientation of WM fiber tracts is valid in the diseased brain. Indeed, complex tissue reorganization by infiltrating lesions could cause the local major eigenvector direction to correspond to nonaxonal tissue organization. However, if specific pathology can be shown to affect the eigenvector distributions in a repeatable manner, it may still be possible to use these directional statistics measures for characterizing diseased tissues.

\section{CONCLUSIONS}

The scatter matrix formalism provides novel information about the distribution of major eigenvector directions. The vector distribution can be quantified using directional statistics and visualized using rose diagrams and directional scatterplots. This approach objectifies the analysis of directional diffusion in pathologic brain tissue, which has previously relied on subjective assessments of directional color maps or similar displays. The explicit quantitation of eigenvector directional distributions promises to find im- portant application in noninvasive tissue characterization and clinical research.

\section{ACKNOWLEDGMENTS}

The authors thank Jee Eun Lee for very helpful support with the DT-MRI image processing. Diffusion encoding direction sets were originally developed by Khader Hasan.

\section{REFERENCES}

1. Basser PJ, Pierpaoli C. Microstructural and physiological features of tissues elucidated by quantitative-diffusion-tensor MRI. Magn Reson Med 1996;111:209-219.

2. Pierpaoli C, Jezzard P, Basser PJ, Barnett A, DiChiro G. Diffusion tensor MR imaging of the human brain. Radiology 1996;201:637-648.

3. Mukherjee P, Bahn MM, Mckinstry RC, Shimony JS, Cull TS, Akbudak E, Snyder AZ, Conturo TE. Differences between gray matter and white matter water diffusion in stroke: diffusion-tensor MR imaging in 12 patients. Radiology 2000;215:211-220.

4. Gauvain KM, Mckinstry RC, Mukherjee P, Perry A, Neil JJ, Kaufman BA, Hayashi RJ. Evaluating pediatric brain tumor cellularity with diffusion-tensor imaging. Am J Roentgenol 2001;177:449-454.

5. Sinha S, Bastin ME, Whittle IR, Wardlaw JM. Diffusion tensor MR imaging of high-grade cerebral gliomas. Am J Neuroradiol 2002;23: $520-527$.

6. Lu S, Ahn D, Johnson G, Cha S. Peritumoral diffusion tensor imaging of high-grade gliomas and metastatic brain tunmors. Am J Neuroradiol 2003;24:937-941.

7. Pajevic S, Pierpaoli C. Color schemes to represent the orientation of anisotropic tissues from diffusion tensor data: application to white matter fiber tract mapping in the human brain. Magn Reson Med 1999; 42:526-540.

8. Mardia KV, Jupp PE. Directional statistics. New York: John Wiley \& Sons; 1999.

9. LeBihan D, Mangin JF, Poupon C, Clark CA, Pappata S, Molko N, Chabriat H. Diffusion tensor imaging: concepts and applications. Magn Reson Med 2001;13:534-546.

10. Hasan KM, Parker DL, Alexander AL. Comparison of gradient encoding schemes for diffusion-tensor MRI. J Magn Reson Imag 2001;13:769780 .

11. Woods RP, Grafton ST, Holmes CJ, Cherry SR, Mazziotta JC. Automated image registration. I. General methods and intrasubject, intramodality validation. J Comput Assist Tomogr 1998;22:139-152.

12. Hasan KM, Basser PJ, Parker DL, Alexander AL. Analytical computation of the eigenvalues and eigenvectors in DT-MRI. J Magn Reson 2001;152:41-47.

13. Wu YC, Field AS, Badie B, Alexander AL. Quantitative analysis of diffusion tensor eigenvectors of white matter infiltration by tumor and edema. In: Proc 11th Annual Meeting ISMRM, Toronto, 2003. p 2076.

14. Jellison BJ, Field AS, Medow J, Lazar M, Salamat MS, Alexander AL. Diffusion tensor imaging of cerebral white matter: a pictorial review of physics, fiber tract anatomy, and tumor imaging patterns. Am J Neuroradiol 2004;25:356-369.

15. Jellison BJ, Wu YC, Field AS, Hasan KM, Alexander AL, Badie B Diffusion tensor metrics for tissue characterization: discriminating vasogenic edema from infiltrating tumor. In: Proc 41st Annual Meeting ASNR, Washington, DC, 2003. p 1154.

16. Basser PJ, Pajevic S. Statistical artifacts in diffusion tensor MRI (DTMRI) caused by background noise. Magn Reson Med 2000;44:41-50.

17. Bingham C. An antipodally symmetric distribution on the sphere. Ann Stat 1974;2:1201-1225.

18. Jones DK, Griffin LD, Alexander DC, Catani M, Horsfield MA, Howard R, Williams SCR. Spatial normalization and averaging of diffusion tensor MRI data sets. NeuroImage 2002;17:592-617.

19. Li TQ. White matter fiber orientation distribution in normal adult brains is not evenly distributed overall. In: Proc 10th Annual Meeting ISMRM, Honolulu, 2002.

20. Hasan KM, Parker DL, Alexander AL. Magnetic resonance water selfdiffusion tensor encoding optimization methods for full brain acquisition. Image Anal Stereol 2002;21:87-96. 